\title{
Light controls light: single molecules as optical switches
}

\author{
Johannes Maier ${ }^{1}$, Martti Pärs ${ }^{1}$, Katja Gräf ${ }^{2}$, Mukundan Thelakkat $^{2}$, and Jürgen Köhler ${ }^{1, *}$ \\ ${ }^{1}$ Spectroscopy of soft Matter, University of Bayreuth, 95447 Bayreuth, Germany \\ ${ }^{2}$ Applied Functional Polymers, University of Bayreuth, 95447 Bayreuth Germany
}

\begin{abstract}
In recent years much attention has been given to design multistate molecular components with functionalities that cover the range from simple switches to logic gates [1-3]. In this regard photochromic molecules, i.e., molecules that can be interconverted between two bistable forms by light, have played an important role. Promising candidates that fulfill obvious demands such as high photochemical/ photophysical stability and high fatigue resistance are compounds of the family of diarylethenes [2,3]. However, a serious drawback of this class of molecules is a low fluorescence quantum yield. Therefore we adapted the strategy developed by Irie and coworkers [2,3], to chemically synthesize complex tailor-made triads consisting of a photochromic dithienylcyclopentene (DCP) unit covalently linked to two peryline bisimide (PBI) molecules that are known as strong fluorophores, see fig. 1 inset top left. This facilitates the combination of high fatigue resistance and high fluorescence quantum yield. Illumination with light in the UV spectral region induces a ringclosure reaction of the DCP and leads to a state with suppressed fluorescence from the PBIs, whereas light in the VIS spectral region yields a ring opening reaction of the DCP and restores the fluorescence from the PBI units. This allowed us to verify functionalities like optical gating and amplifying, yet where the electrons have been replaced by photons as signal carriers [4-6], see fig.1.
\end{abstract}

Switching of photochromic molecules at the single molecule level has been demonstrated before [7], yet in those experiments no distinction was made between deliberate switching and unavoidable blinking of single molecules. The switching process is manifested as a finite delay between the onset of the fluorescence and the onset of the VIS laser. For a sequence of 8800 switching events the distribution of delay times can be reasonably well approximated as an exponential decay, in agreement with the expectation for a switching process that obeys Poissonian statistics [8]. From a statistical analysis, that takes results from reference experiments performed on the same triad without switching into account, we determined the positive predictive value $\mathrm{p}(\mathrm{S} \mid+)$ for deliberate light-induced switching. In words: The conditional probability that the triad indeed has switched given that we observed a finite delay for the onset of the fluorescence. For 15 triads studied the average of this probability amounts to $0.8 \pm 0.1$. In the best cases it reached about $96 \%$,

* Corresponding author: juergen.koehler@uni-bayreuth.de 
insufficient for optical memories with single triads, yet exceptionally good for superresolution microscopy.

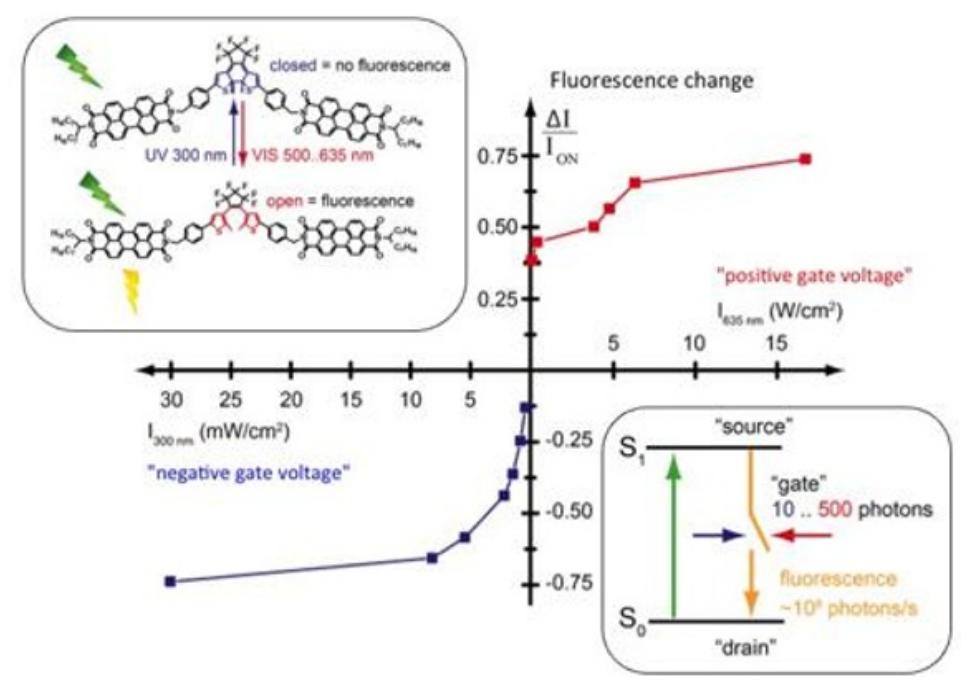

Fig. 1. Relative change of the fluorescence of the photochromic triads as a function of the intensity of the UV/VIS conversion beams. The flow of (many) fluorescence photons from the PBI units can be controlled by (few) UV and VIS photons that induce the photocyclization / photocycloreversion reactions of the photochromic DCP unit, respectively.

\section{References}

1. B. L. Feringa, Molecular Switches (Wiley-VCH, Weinheim, 2001)

2. M. Irie, Chem. Rev., 100, 1685 (2000)

3. M. Irie, T. Fukaminato, K. Matsuda, S. Kobatake, Chem. Rev. 114, 12174 (2014)

4. M. Pärs, C. C. Hofmann, K. Willinger, P. Bauer, M. Thelakkat, J. Köhler, Ang. Chem. Int. Ed., 50, 11405 (2011)

5. M. Pärs, K. Gräf, P. Bauer, M. Thelakkat, J. Köhler, Ap. Phys. Lett., 103, 221115 (2013)

6. M. Pärs, M. Gradmann, K. Gräf, P. Bauer, M. Thelakkat, J. Köhler, Scientific Reports, 4, 4316 (2014)

7. M. Irie, T. Fukaminato, T. Sasaki, N. Tamai, T. Kawai, Nature, 420, 759 (2002)

8. J. Maier, M. Pärs, T. Weller, M. Thelakkat, J. Köhler, Scientific Reports, 7, 41739 (2017) 